\title{
New topological structures of Skyrme theory: baryon number and monopole number
}

\author{
Y. M. Cho ${ }^{1,2,3, a}$, Kyoungtae Kimm ${ }^{4}$, J. H. Yoon ${ }^{5}$, Pengming Zhang ${ }^{1}$ \\ ${ }^{1}$ Institute of Modern Physics, Chinese Academy of Science, Lanzhou 730000, China \\ ${ }^{2}$ Konkuk University, Administration Building 310-4, Seoul 143-701, Korea \\ ${ }^{3}$ School of Physics and Astronomy, Seoul National University, Seoul 151-742, Korea \\ ${ }^{4}$ Faculty of Liberal Education, Seoul National University, Seoul 151-747, Korea \\ ${ }^{5}$ Department of Physics, Konkuk University, Seoul 143-701, Korea
}

Received: 1 March 2016 / Accepted: 26 January 2017 / Published online: 9 February 2017

(c) The Author(s) 2017. This article is published with open access at Springerlink.com

\begin{abstract}
Based on the observation that the skyrmion in Skyrme theory can be viewed as a dressed monopole, we show that the skyrmions have two independent topology, the baryon topology $\pi_{3}\left(S^{3}\right)$ and the monopole topology $\pi_{2}\left(S^{2}\right)$. With this we propose to classify the skyrmions by two topological numbers $(m, n)$, the monopole number $m$ and the shell (radial) number $n$. In this scheme the popular (non spherically symmetric) skyrmions are classified as the $(m, 1)$ skyrmions but the spherically symmetric skyrmions are classified as the $(1, n)$ skyrmions, and the baryon number $B$ is given by $B=m n$. Moreover, we show that the vacuum of the Skyrme theory has the structure of the vacuum of the SineGordon theory and QCD combined together, which can also be classified by two topological numbers $(p, q)$. This puts the Skyrme theory in a totally new perspective.
\end{abstract}

\section{Introduction}

The Skyrme theory has played an important role in physics. It has been proposed as an effective field theory of pion physics in strong interaction where the baryons appear as the skyrmions, topological solitons made of pions [1-4]. This view has been very successful, and the rich topological structure of the theory has advanced our understanding of the extended objects greatly [5-7].

The construction of skyrmions as nuclei has a long history. A novel way to obtain non-spherically symmetric multiskyrmions was developed based on the rational map, and the solutions have been associated with and compared to real nuclei $[8,9]$. And a systematic approach to construct the skyrmions with large baryon number numerically which have the shell strucutre has been developed [10-13]. This,

a e-mail: ymcho7@konkuk.ac.kr with the improved computational power has made people construct skyrmions with the baryon number up to 108 [14].

With the new development the Skyrme theory have had a remarkable progress recently. It has been able to provide a quantitative understanding of the spectrum of rotational excitations of carbon-12, including the excitation the Hoyle state which is essential for the generation of heavy nuclear elements in early universe [15-17]. And the spin-orbit interaction which is essential for the magic number of nuclei is investigated within the framework of Skyrme theory [18]. Moreover, a method to reduce the binding energy of skyrmions to a realistic level to improve the Skyrme model has been developed $[19,20]$. So by now in principle one could construct all nuclei as multi-baryon skyrmions and discuss the phenomenology of nuclear physics, although the experimental confirmation of the theory is still in dispute.

But the Skyrme theory has multiple faces. In addition to the well known skyrmions it has the (helical) baby skyrmion and the Faddeev-Niemi knot. Most importantly, it has the monopole which plays the fundamental role [21-23]. In this view all finite energy topological objects in the theory could be viewed either as dressed monopoles or as confined magnetic flux of the monopole-antimonopole pair. The skyrmion can be viewed as a dressed monopole, the baby skyrmion as a magnetic vortex created by the monopole-antimonopole pair infinitely separated apart, and the Faddeev-Niemi knot as a twisted magnetic vortex ring made of the helical baby skyrmion. This confirms that the theory can be interpreted as a theory of monopole in which the magnetic flux of the monopoles is confines and/or screened.

The fact that the skyrmion is closely related to the monopole has been appreciated for a long time. It has been well known that the skyrmions could actually be viewed as the monopoles regularized to have finite energy [21-23]. 
In fact it has been well appreciated that the rational map which plays the crucial role in the construction of the multiskyrmions is exactly the $\pi_{2}\left(S^{2}\right)$ mapping which provides the monopole quantum number [10]. Nevertheless the skyrmions have always been classified by the baryon number given by $\pi_{3}\left(S^{3}\right)$, not by the monopole number $\pi_{2}\left(S^{2}\right)$. This was puzzling.

The purpose of this paper is twofold. We first show that the skyrmions have two topology, the baryon topology and the monopole topology, so that they are classified by two topological numbers, the baryon number $B$ and the monopole number $M$. Moreover, we show that the baryon number can be replaced by the radial (shell) number which describes the $\pi_{1}\left(S^{1}\right)$ topology of radial excitation of multi-skyrmions. This is based on the observation that the SU(2) space $S^{3}$ has the Hopf fibering $S^{3} \simeq S^{2} \times S^{1}$ and that the Skyrme theory is described by two variables which naturally represent the $S^{2}$ and $S^{1}$ manifolds.

Second, we show that the vacuum of the Skyrme theory has the structure of the vacuum of the Sine-Gordon theory and QCD combined together, which can also be classified by two topological numbers $(p, q)$.

The paper is organized as follows. In Sect. 2 we briefly review the old skyrmions for later purpose. In Sect. 3 we show that the skyrmions carry two topological numbers, the baryon number $b$ and the monopole number $m$. Moreover, we show that the baryon number can be replaced by the radial (shell) number $n$, so that they can be classified by $(m, n)$. In this scheme the baryon number is given by $b=m n$. In Sect. 4 we discuss the vacuum structure of the Skyrme theory, and show that it has the structure of the vacuum of the Sine-Gordon theory combined with the vacuum of the SU(2) QCD. This tells that it can be classified by two topological numbers denoted by $(p, q)$, where $p$ and $q$ represent the $\pi_{1}\left(S^{1}\right)$ topology of the Sine-Gordon theory and the $\pi_{3}\left(S^{2}\right)$ topology of QCD vacuum. Finally in Sect. 5 we discuss the physical implications of our results.

\section{Skyrme theory: a review}

To see this let $\omega$ and $\hat{n}\left(\hat{n}^{2}=1\right)$ be the massless sigma field and the normalized pion field in Skyrme theory, and consider the Skyrme Lagrangian

$$
\begin{aligned}
\mathcal{L}= & \frac{\kappa^{2}}{4} \operatorname{tr} L_{\mu}^{2}+\frac{\alpha}{32} \operatorname{tr}\left(\left[L_{\mu}, L_{\nu}\right]\right)^{2} \\
= & -\frac{\kappa^{2}}{4}\left[\frac{1}{2}\left(\partial_{\mu} \omega\right)^{2}+2 \sin ^{2} \frac{\omega}{2}\left(\partial_{\mu} \hat{n}\right)^{2}\right] \\
& -\frac{\alpha}{8} \sin ^{2} \frac{\omega}{2}\left[\left(\partial_{\mu} \omega\right)^{2}\left(\partial_{\nu} \hat{n}\right)^{2}-\left(\partial_{\mu} \omega \partial_{\nu} \omega\right)\left(\partial_{\mu} \hat{n}\right) \cdot\left(\partial_{\nu} \hat{n}\right)\right] \\
& +\frac{\alpha}{4} \sin ^{4} \frac{\omega}{2}\left(\partial_{\mu} \hat{n} \times \partial_{\nu} \hat{n}\right)^{2},
\end{aligned}
$$

$L_{\mu}=U \partial_{\mu} U^{\dagger}$

$U=\exp \left(\frac{\omega}{2 i} \vec{\sigma} \cdot \hat{n}\right)=\cos \frac{\omega}{2}-i(\vec{\sigma} \cdot \hat{n}) \sin \frac{\omega}{2}$,

where $\kappa$ and $\alpha$ are the coupling constants. Notice that $\hat{n}$ and $\omega$ naturally describe the $S^{2}$ and $S^{1}$ manifold. With

$U=\sigma-i \vec{\sigma} \cdot \vec{\pi}$

$\sigma=\cos \frac{\omega}{2}, \quad \vec{\pi}=\hat{n} \sin \frac{\omega}{2}, \quad\left(\sigma^{2}+\vec{\pi}^{2}=1\right)$,

the Lagrangian (1) has the familiar form

$$
\begin{aligned}
\mathcal{L}= & -\frac{\kappa^{2}}{2}\left(\left(\partial_{\mu} \sigma\right)^{2}+\left(\partial_{\mu} \vec{\pi}\right)^{2}\right) \\
& -\frac{\alpha}{4}\left(\left(\partial_{\mu} \sigma \partial_{\nu} \vec{\pi}-\partial_{\nu} \sigma \partial_{\mu} \vec{\pi}\right)^{2}+\left(\partial_{\mu} \vec{\pi} \times \partial_{\nu} \vec{\pi}\right)^{2}\right) \\
& +\frac{\lambda}{4}\left(\sigma^{2}+\vec{\pi}^{2}-1\right),
\end{aligned}
$$

where $\lambda$ is a Lagrange multiplier. In this form $\sigma$ and $\vec{\pi}$ represent the sigma and pion fields, so that the Skyrme theory describes the pion physics.

The Lagrangian has a hidden $U(1)$ gauge symmetry as well as a global $\mathrm{SU}(2)_{L} \times \mathrm{SU}(2)_{R}$ symmetry [22,23]. The global SU(2) symmetry is obvious, but the hidden $U$ (1) symmetry is not. It comes from the fact that $\hat{n}$ has an invariant subgroup $U(1)$. To see this, we reparametrize $\hat{n}$ by the $\mathrm{CP}^{1}$ field $\xi$,

$\vec{n}=\xi^{\dagger} \vec{\sigma} \xi, \quad \xi^{\dagger} \xi=1$,

and find that under the $U(1)$ gauge transformation of $\xi$

$\xi \rightarrow \exp (i \theta(x)) \xi$,

$\hat{n}$ (and $\partial_{\mu} \hat{n}$ ) remains invariant. Now, we introduce the composite gauge potential $B_{\mu}$ and the covariant derivative $D_{\mu}$ which transforms gauge covariantly under (5) by

$B_{\mu}=-i \xi^{\dagger} \partial_{\mu} \xi, \quad D_{\mu} \xi=\left(\partial_{\mu}-i B_{\mu}\right) \xi$.

With this we have the following identities,

$$
\begin{aligned}
\left(\partial_{\mu} \hat{n}\right)^{2}=4\left|D_{\mu} \xi\right|^{2} & \\
\partial_{\mu} \hat{n} \times \partial_{\nu} \hat{n} & =-2 i\left[\left(\partial_{\mu} \xi^{\dagger}\right)\left(\partial_{\nu} \xi\right)-\left(\partial_{\mu} \xi^{\dagger}\right)\left(\partial_{\nu} \xi\right)\right] \hat{n} \\
& =2 G_{\mu \nu} \hat{n}, \quad G_{\mu \nu}=\partial_{\mu} B_{\nu}-\partial_{\nu} B_{\mu} .
\end{aligned}
$$

Furthermore, with the Fierz' identity

$\sigma_{i j}^{a} \sigma_{k l}^{a}=2 \delta_{i l} \delta_{j k}-\delta_{i j} \delta_{k l}$,

we have

$$
\begin{aligned}
\partial_{\mu} \hat{n} \cdot \partial_{\nu} \hat{n}= & 2 \partial_{\mu}\left(\xi_{i}^{\dagger} \xi_{j}\right) \partial_{\nu}\left(\xi_{j}^{\dagger} \xi_{i}\right) \\
= & 2\left[\left(\partial_{\mu} \xi^{\dagger} \xi\right)\left(\partial_{\nu} \xi^{\dagger} \xi\right)+\left(\partial_{\mu} \xi^{\dagger}\right)\left(\partial_{\nu} \xi\right)\right. \\
& \left.+\left(\partial_{\nu} \xi^{\dagger}\right)\left(\partial_{\mu} \xi\right)+\left(\xi^{\dagger} \partial_{\mu} \xi\right)\left(\xi^{\dagger} \partial_{\nu} \xi\right)\right] \\
= & 2\left[\left(D_{\mu} \xi\right)^{\dagger}\left(D_{\nu} \xi\right)+\left(D_{\nu} \xi\right)^{\dagger}\left(D_{\mu} \xi\right)\right] .
\end{aligned}
$$


From this we can express (1) by

$$
\begin{aligned}
\mathcal{L}= & -\frac{\kappa^{2}}{4}\left[\frac{1}{2}\left(\partial_{\mu} \omega\right)^{2}+8 \sin ^{2} \frac{\omega}{2}\left|D_{\mu} \xi\right|^{2}\right] \\
& -\frac{\alpha}{2} \sin ^{2} \frac{\omega}{2}\left[\left(\partial_{\mu} \omega\right)^{2}\left|D_{\mu} \xi\right|^{2}-\left(\partial_{\mu} \omega \partial_{\nu} \omega\right)\left(D_{\mu} \xi\right)^{\dagger}\left(D_{\nu} \xi\right)\right] \\
& -\alpha \sin ^{4} \frac{\omega}{2} G_{\mu \nu}^{2},
\end{aligned}
$$

which is explicitly invariant under the $U(1)$ gauge transformation (5). So replacing $\hat{n}$ by $\xi$ in the Lagrangian we can make the hidden $U(1)$ gauge symmetry explicit. In this form the Skyrme theory becomes a self-interacting $U(1)$ gauge theory of $\mathrm{CP}^{1}$ field coupled to a massless scalar field.

From (1) we have the following equations of motion [2123]

$$
\begin{aligned}
& \partial^{2} \omega-\sin \omega\left(\partial_{\mu} \hat{n}\right)^{2}+\frac{\alpha}{8 \kappa^{2}} \sin \omega\left(\partial_{\mu} \omega \partial_{\nu} \hat{n}-\partial_{\nu} \omega \partial_{\mu} \hat{n}\right)^{2} \\
& +\frac{\alpha}{\kappa^{2}} \sin ^{2} \frac{\omega}{2} \partial_{\mu}\left[\left(\partial_{\mu} \omega \partial_{\nu} \hat{n}-\partial_{\nu} \omega \partial_{\mu} \hat{n}\right) \cdot \partial_{\nu} \hat{n}\right] \\
& -\frac{\alpha}{\kappa^{2}} \sin ^{2} \frac{\omega}{2} \sin \omega\left(\partial_{\mu} \hat{n} \times \partial_{\nu} \hat{n}\right)^{2}=0, \\
& \partial_{\mu}\left\{\sin ^{2} \frac{\omega}{2} \hat{n} \times \partial_{\mu} \hat{n}+\frac{\alpha}{4 \kappa^{2}} \sin ^{2} \frac{\omega}{2}\right. \\
& \quad \times\left[\left(\partial_{\nu} \omega\right)^{2} \hat{n} \times \partial_{\mu} \hat{n}-\left(\partial_{\mu} \omega \partial_{\nu} \omega\right) \hat{n} \times \partial_{\nu} \hat{n}\right] \\
& \left.\quad+\frac{\alpha}{\kappa^{2}} \sin ^{4} \frac{\omega}{2}\left(\hat{n} \cdot \partial_{\mu} \hat{n} \times \partial_{\nu} \hat{n}\right) \partial_{\nu} \hat{n}\right\}=0 .
\end{aligned}
$$

It has two interesting limits. First, in the spherically symmetric limit

$\omega=\omega(r), \quad \hat{n}= \pm \hat{r}$,

it is reduced to

$$
\begin{gathered}
\frac{\mathrm{d}^{2} \omega}{\mathrm{d} r^{2}}+\frac{2}{r} \frac{\mathrm{d} \omega}{\mathrm{d} r}-\frac{2 \sin \omega}{r^{2}}+\frac{2 \alpha}{\kappa^{2}}\left[\frac{\sin ^{2}(\omega / 2)}{r^{2}} \frac{\mathrm{d}^{2} \omega}{\mathrm{d} r^{2}}\right. \\
\left.+\frac{\sin \omega}{4 r^{2}}\left(\frac{\mathrm{d} \omega}{\mathrm{d} r}\right)^{2}-\frac{\sin \omega \sin ^{2}(\omega / 2)}{r^{4}}\right]=0 .
\end{gathered}
$$

Adopting the spherically symmetric ansatz (12) imposing the boundary condition

$\omega(0)=2 \pi, \quad \omega(\infty)=0$,

we obtain the original skyrmion solution solving (13) which has the finite energy [1-3]. It carries the baryon number

$$
\begin{aligned}
B & =-\frac{1}{8 \pi^{2}} \int \epsilon_{i j k} \partial_{i} \omega\left[\hat{n} \cdot\left(\partial_{j} \hat{n} \times \partial_{k} \hat{n}\right)\right] \sin ^{2} \frac{\omega}{2} \mathrm{~d}^{3} r \\
& =1
\end{aligned}
$$

which represents the non-trivial homotopy $\pi_{3}\left(S^{3}\right)$ defined by $U$ in (1).

Second, when

$\omega=(2 n+1) \pi$, the equation is reduced to

$\hat{n} \times \partial^{2} \hat{n}+\frac{\alpha}{\kappa^{2}}\left(\partial_{\mu} H_{\mu \nu}\right) \partial_{\nu} \hat{n}=0$,

$H_{\mu \nu}=\hat{n} \cdot\left(\partial_{\mu} \hat{n} \times \partial_{\nu} \hat{n}\right)=\partial_{\mu} C_{\nu}-\partial_{\nu} C_{\mu}$,

where $C_{\mu}$ is the magnetic potential of $H_{\mu \nu}$. This is the central equation of Skyrme theory which allows the monopole, the baby skyrmion, the twisted magnetic vortex, and the knot [21-23]. In fact (17) has the monopole solution [21-23]

$\hat{n}= \pm \hat{r}$.

which carries the magnetic charge

$M=\frac{ \pm 1}{8 \pi} \int \epsilon_{i j k}\left[\hat{r} \cdot\left(\partial_{i} \hat{r} \times \partial_{j} \hat{r}\right)\right] \mathrm{d} \sigma_{k}= \pm 1$,

which represents the homotopy $\pi_{2}\left(S^{2}\right)$ defined by $\hat{n}$.

Notice that, with (16) the Skyrme Lagrangian becomes the Skyrme-Faddeev Lagrangian,

$\mathcal{L} \rightarrow-\frac{\kappa^{2}}{2}\left(\partial_{\mu} \hat{n}\right)^{2}-\frac{\alpha}{4}\left(\partial_{\mu} \hat{n} \times \partial_{\nu} \hat{n}\right)^{2}$,

whose equation of motion is given by (17). This tells that the Skyrme-Faddeev theory is an essential ingredient, the back bone, of the Skyrme theory. As importantly, this reveals the "missing link" between Skyrme theory and QCD. This is because we can derive the Skyrme-Faddeev Lagrangian directly from QCD, which shows that the two theories are related by the Skyrme-Faddeev Lagrangian as the common denominator [21-23].

Solving (11) for multi-skyrmions numerically with (14) choosing $\hat{n}$ to describe an arbitrary rational map $\pi_{2}\left(S^{2}\right)$, one can obtain the well known (non spherically symmetric) multiskyrmion solutions whose baryon number is given by the rational map number of $\hat{n}$ numerically [8-11]. Some of these solutions are copied from Ref. [10] in Fig. 1.

In addition to these popular solutions we have other spherically symmetric multi-skyrmions. To obtain them notice that, although the $\mathrm{SU}(2)$ matrix $U$ is periodic in $\omega$ variable by $4 \pi$, $\omega$ itself can take any value from $-\infty$ to $+\infty$. So we can obtain the spherically symmetric solution of (13) with the boundary condition

$\omega(0)=2 \pi n, \quad \omega(\infty)=0$,

with an arbitrary integer $n$ [1-7]. Clearly they have the baryon number

$$
\begin{aligned}
B & =\frac{1}{8 \pi^{2}} \int \epsilon_{i j k} \partial_{i} \omega\left[\hat{r} \cdot\left(\partial_{j} \hat{r} \times \partial_{k} \hat{r}\right)\right] \sin ^{2} \frac{\omega}{2} \mathrm{~d}^{3} r \\
& =\frac{1}{\pi} \int \sin ^{2} \frac{\omega}{2} \mathrm{~d} \omega=n .
\end{aligned}
$$

This means that the baryon number is given by the winding number $\pi_{1}\left(S^{1}\right)$ of $\omega$, which is determined by the boundary condition (21). In Fig. 2 we present the spherically symmetric skyrmions for $n=1,2,3,4,5,6,7$. 


\section{Baryon number versus monopole number}

The contrast between the two sets of solutions shown in Figs. 1 and 2 is unmistakable, but this is not just in the appearence. They have a fundamental difference. Clearly the baryon number of the skyrmions shown in Fig. 1 is given by the rational map $\pi_{2}\left(S^{2}\right)$ defined by $\hat{n}$ [8-13]. Moreover, the rational map $\pi_{2}\left(S^{2}\right)$ of $\hat{n}$ in the non spherically symmetric solutions is precisely the monopole topology of the skyrmion which determines the monopole number $M$ [21-23]

$M=\frac{1}{8 \pi} \int \epsilon_{i j k}\left[\hat{n} \cdot\left(\partial_{i} \hat{n} \times \partial_{j} \hat{n}\right)\right] \mathrm{d} \sigma_{k}=m$.

And clearly this monopole number is different from the baryon number given by the $\pi_{3}\left(S^{3}\right)$ topology.

However, the baryon number of the skyrmions shown in Fig. 2 is given by the winding number $\pi_{1}\left(S^{1}\right)$ of $\omega$ which has nothing to do with the rational map of $\hat{n}$. Moreover, the

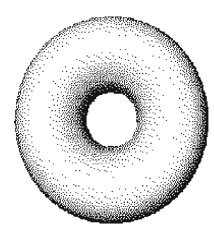

a

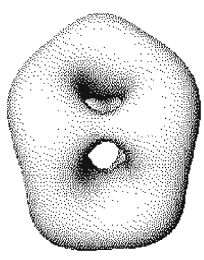

d

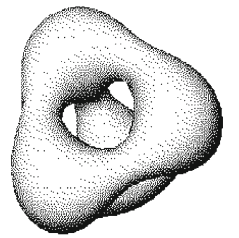

b

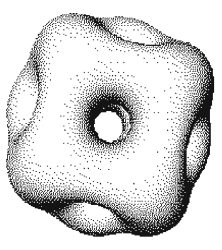

$\mathbf{e}$

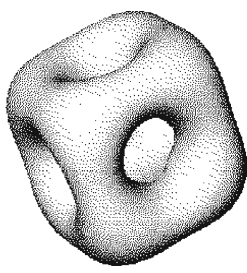

c

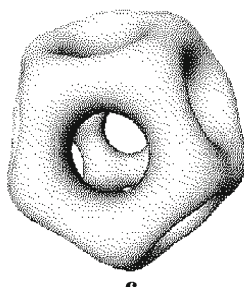

f
Fig. 1 The well known (non spherically symmetric) numerical multiskyrmion solutions with baryon number $2,3,4,5,6$, and 7 , copied from Ref. [10]

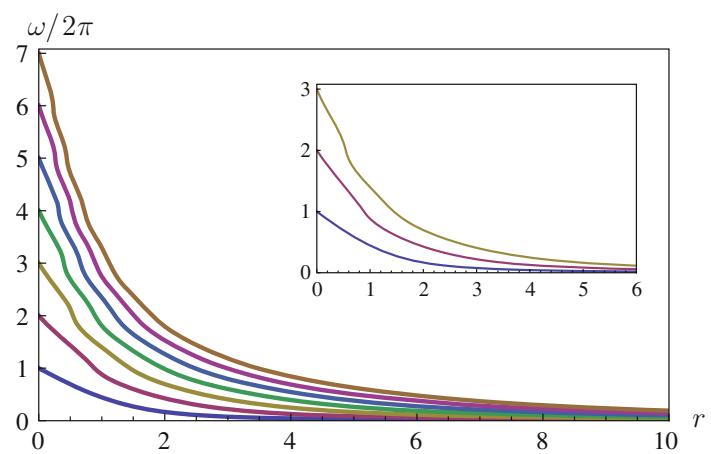

Fig. 2 The spherically symmetric solutions with baryon number $1,2,3,4,5,6,7$, which should be contrasted with the popular multiskyrmion solutions shown in Fig. 1 monopole number of these solutions given by the rational map $\pi_{2}\left(S^{2}\right)$ of $\hat{n}$ is

$M=\frac{1}{8 \pi} \int \epsilon_{i j k}\left[\hat{r} \cdot\left(\partial_{i} \hat{r} \times \partial_{j} \hat{r}\right)\right] \mathrm{d} \sigma_{k}=1$.

This tells that the baryon number and the monopole number of these solutions are different.

This shows that the skyrmions actually have two topological numbers $B$ and $M$ which are in principle different. But this was not evident in the popular skyrmion solutions because they have $B=M$. But obviously the spherically symmetric solutions have two topological numbers, the baryon number $B=n$ and the monopole number $M=1[1-3,8]$. This proves that the skyrmions do have two topology denoted by $(b, m)$, the $\pi_{3}\left(S^{3}\right)$ which describes the baryon number $b$ and the $\pi_{2}\left(S^{2}\right)$ which describes the monopole number $m$. But so far this important point has been completely neglected.

Moreover, the integer $n$ in (21) has another meaning. It describes the $\pi_{1}\left(S^{1}\right)$ topology of the angular variable $\omega$ which depends only on the radial coordinate $r$. Moreover, it could be viewed as the radial, or more properly the shell quantum number, since the spherically symmetric solutions can be viewed as the generalization of the original skyrmion which has radially excited shells where $n$ describes the number of the shells. This implies that we could also classify the skyrmions by $(m, n)$, the $\pi_{2}\left(S^{2}\right)$ topology of $\hat{n}$ and $\pi_{1}\left(S^{1}\right)$ topology of $\omega$.

In this scheme the well known (non spherically symmetric) numerical multi-skyrmion solutions shown in the earlier works $[9,10]$ become the $(m, 1)$ skyrmions, but those shown in Fig. 2 become the $(1, n)$ skyrmions. This strongly implies that the baryon number is made of two parts, the $\pi_{2}\left(S^{2}\right)$ of $\hat{n}$ and $\pi_{1}\left(S^{1}\right)$ of $\omega$.

To amplify this point we notice the followings. First, the $S^{3}$ space (both the real space and the target space) in $\pi_{3}\left(S^{3}\right)$ admits the Hopf fibering $S^{3} \simeq S^{2} \times S^{1}$. Second, the two variables $\hat{n}$ and $\omega$ of the Skyrme theory naturally represent $S^{2}$ and $S^{1}$. So the baryon number of the $(m, n)$ skyrmion is given by

$$
\begin{aligned}
B & =-\frac{1}{8 \pi^{2}} \int \epsilon_{i j k} \partial_{i} \omega\left[\hat{n} \cdot\left(\partial_{j} \hat{n} \times \partial_{k} \hat{n}\right)\right] \sin ^{2} \frac{\omega}{2} \mathrm{~d}^{3} r \\
& =-\frac{1}{8 \pi^{2}} \int \partial_{i} \omega\left[\hat{n} \cdot\left(\partial_{j} \hat{n} \times \partial_{k} \hat{n}\right)\right] \sin ^{2} \frac{\omega}{2} \mathrm{~d} x^{i} \wedge \mathrm{d} x^{j} \wedge \mathrm{d} x^{k} \\
& =-\frac{1}{8 \pi^{2}} \int \sin ^{2} \frac{\omega}{2} d \omega \times \epsilon_{i j k}\left[\hat{n} \cdot\left(\partial_{i} \hat{n} \times \partial_{j} \hat{n}\right)\right] \mathrm{d} \Sigma_{k} \\
& =\frac{n}{8 \pi} \int \epsilon_{i j k}\left[\hat{n} \cdot\left(\partial_{i} \hat{n} \times \partial_{j} \hat{n}\right)\right] \mathrm{d} \Sigma_{k}=m n,
\end{aligned}
$$

where $\mathrm{d} \Sigma_{k}=\epsilon_{i j k} \mathrm{~d} x^{i} \wedge \mathrm{d} x^{j} / 2$. Clearly the last integral is topologically equivalent to (23), which assures the last equality. This shows that the baryon number of the skyrmion can be decomposed to the monopole number and the shell number. 
Obviously both $(m, 1)$ and $(1, n)$ skyrmions are the particular examples of this.

At this point it must be emphasized that the shell number $n$ has first been introduced by Manton and Piette [11]. They have noticed that the skyrmions can be generalized to have the multiple shell structure which can be expressed by the shell number. Moreover, they have shown that this shell structure is very useful to construct the multi-skyrmions which have a large baryon number even for the non spherically symmetric skyrmions.

What we propose here is that the baryon number can be decomposed to the monopole number and the shell number, and that this shell number could be interpreted to represent an independent topology of the skyrmion. This follows from the fact that the skyrmions have two independent topology, the monopole topology $\pi_{2}\left(S^{2}\right)$ and the baryon topology $\pi_{3}\left(S^{3}\right)$. Given this fact, the natural question is how they are related. The answer is that the baryon topology is made of the monopole topology and the shell topology, and the baryon number is given by the product of the monopole number and the shell number.

This is based on two facts. First, the Skyrme theory is made of two variables, the $S^{2}$ variable $\hat{n}$ which represents the $\pi_{2}\left(S^{2}\right)$ topology and the $S^{1}$ variable $\omega$ which represents the shell topology $\pi_{1}\left(S^{1}\right)$. Second, the baryon topology is described by both $\omega$ and $\hat{n}$, but the monopole topology is described only by $\hat{n}$. So it becomes only natural that $\omega$ changes the monopole topology to the baryon topology, adding the shell structure to the monopole topology. It is this separation of the roles of the two variables which allows us to replace the baryon topology with the shell topology in Skyrme theory. Obviously this is best demonstrated in the spherically symmetric skyrmions.

An interesting feature of the spherically symmetric solutions is that whenever the curve passes through the values $\omega=2 \pi n$, it become a bit steeper. There is a good reason why this is so. As we will see these points are the vacua of the theory, and the steep slopes shows that the energy likes to be concentrated around these vacua. So these steep slopes are not an irregularity, but just what is expected.

We can easily calculate the energy of the spherically symmetric solutions from [23]

$$
\begin{aligned}
E= & \frac{\pi \kappa^{2}}{2} \int_{0}^{\infty}\left\{\left(r^{2}+\frac{2 \alpha}{\kappa^{2}} \sin ^{2} \frac{\omega}{2}\right)\left(\frac{\mathrm{d} \omega}{\mathrm{d} r}\right)^{2}\right. \\
& \left.+8\left(1+\frac{\alpha}{2 \kappa^{2} r^{2}} \sin ^{2} \frac{\omega}{2}\right) \sin ^{2} \frac{\omega}{2}\right\} \mathrm{d} r \\
= & \pi \sqrt{\alpha} \kappa \int_{0}^{\infty}\left[x^{2}\left(\frac{\mathrm{d} \omega}{\mathrm{d} x}\right)^{2}+8 \sin ^{2} \frac{\omega}{2}\right] \mathrm{d} x,
\end{aligned}
$$

where $x=(\kappa / \sqrt{\alpha}) r$ is a dimensionless variable. The result is shown in Fig. 3. Numerically the baryon number dependence of the energy is given by [5-7]

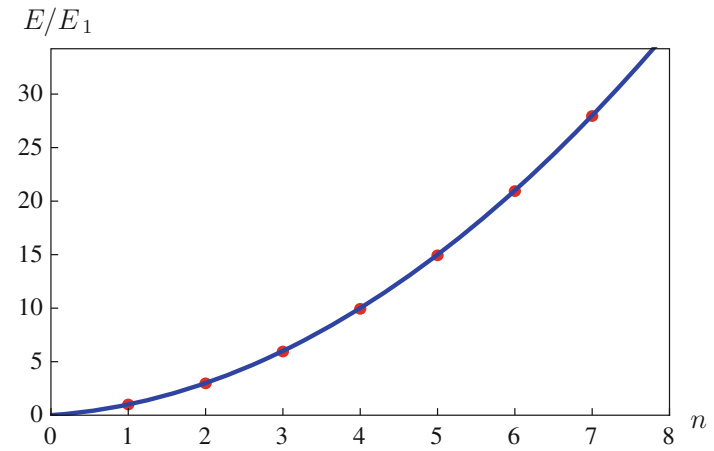

Fig. 3 The energy of the spherically symmetric solutions with baryon number 1,2,3,4,5,6,7. The numerical fit (the blue curve) and the $n(n+$ 1) $E_{1} / 2$ curve (the green curve) are almost indistinguishable

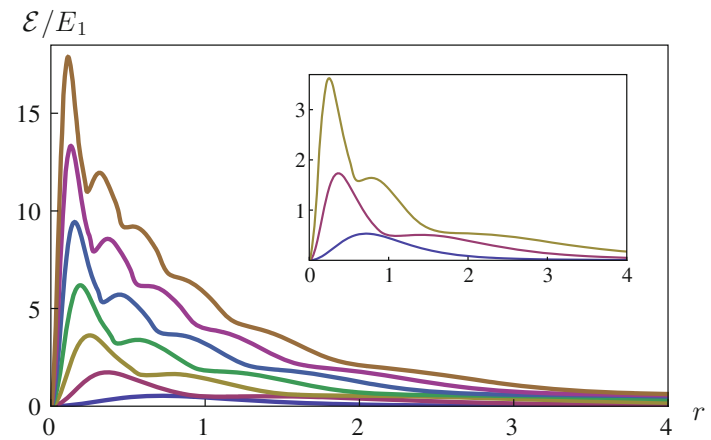

Fig. 4 The energy density of the spherically symmetric skyrmions with baryon number $1,2,3,4,5,6,7$

$E_{n} \simeq \frac{n(n+1)}{2} E_{1}$

This could be understood as follows. Roughly speaking, the kinetic energy (first part) and the potential energy (second part) of (26) become proportional to $n^{2}$ and $n$, and the two terms have equal contribution due to the equipartition of energy. But the truth is more complicated than this, and we need a mathematical explanation of this.

The energy density of the solutions is shown in Fig. 4. The $B=n$ solution has $n$ local maxima, which tells that it is made of $n$ unit skyrmions which make spherical shells. Moreover, as we have remarked the shells are located at the vacuum points $\omega=2 \pi n$.

Of course, these spherically symmetric skyrmions are precisely the multi-skyrmions that Skyrme originally proposed as nuclei which have baryon number larger than one [1-7]. But they become unstable and can decay to the lower energy skyrmions, because the energy $E_{n}$ gets bigger than the $n$ sum of $E_{1}$. This is not so for the popular (non spherically symmetric) multi-skyrmions which have positive binding energy. Because of this the spherically symmetric skyrmions have been dismissed as uninteresting. 
But our analysis makes them more interesting. First of all, they demonstrate that skyrmions actually have two topological numbers, the baryon number and the monopole number, which are different. Moreover, they show that the skyrmions can be made to have the shell structure. As importantly, they tell that the shell number, together with the monopole number, determines the baryon number.

Clearly the shell structure can also be implemented to the $(m, 1)$ skyrmions shown in Fig. 1. To see this we generalize the boundary condition (21), keeping the rational map number $m$ of $\hat{n}$ the same but requiring [11]

$$
\begin{gathered}
\omega\left(r_{k}\right)=2 \pi k, \quad(k=0,1,2, \ldots n), \\
r_{0}=0<r_{1}\left\langle\cdots \left\langle r_{n}=\infty .\right.\right.
\end{gathered}
$$

With this we could find the $(m, n)$ skyrmion numerically minimizing the energy varying $r_{k}(k=1,2, \ldots, n-1)$. This way we can add the shell structure and the shell number to the $(m, 1)$ skyrmion.

Now, one might ask about the stability of the $(m, n)$ skyrmions. In general they may not be stable. For example, the quadratic dependence of the topological number $n$ of the energy (27) makes $(1, n)$ skyrmions energetically unstable. On the other hand, even when they decay, the topology of the solution must not change. In other words the baryon number and the monopole number must be conserved. This, together with $B=m n$, tells that the shell number should also be conserved. From this we conclude that, when an $(m, n)$ skyrmion decays to $\left(m_{1}, n_{1}\right)$ and $\left(m_{2}, n_{2}\right)$ skyrmions, we must have $n=n_{1}+n_{2}$ and $m=m_{1}+m_{2}$. This, of course, is what is expected.

The above discussion raises another deep question. As we have remarked, when $\omega=(2 n+1) \pi$ the Skyrme theory has knot solutions described by $\hat{n}$ whose topology is given by $\pi_{3}\left(S^{2}\right)$, in addition to the skyrmion solutions [21-23]. If so, can we dress the knots with $\omega$ to provide a new type of shell structure, and extend the knots to have two quantum numbers $\pi_{1}\left(S^{1}\right)$ and $\pi_{3}\left(S^{2}\right)$ ? This is a mind boggling question.

\section{Multiple vacua of Skyrme theory}

Now we show that the Skyrme theory in fact has another very important topological structure, the topologically different multiple vacua. To see this, notice that (11) has the solution

$\omega=2 \pi p, \quad(p ;$ integer $)$,

independent of $\hat{n}$. And obviously this is the vacuum solution.

This tells that the Skyrme theory has multiple vacua classified by the integer $p$ which is similar to the Sine-Gordon theory. But unlike the Sine-Gordon theory, here we have the multiple vacua without any potential. Moreover, the above discussion tells that the spherically symmetric skyrmions connect and occupy the $p+1$ adjacent vacua. This means that we can connect all vacua with the spherically symmetric skyrmions. Of course, one could introduce such vacua in Skyrme theory introducing a potential term in the Lagrangian [24]. This is not what we are doing here. We have these vacua without any potential.

But this is not the end of the story. To see this notice that (29) becomes the vacuum independent of $\hat{n}$. This means that $\hat{n}$ can add the $\pi_{3}\left(S^{2}\right)$ topology to each of the multiple vacua classified by another integer $q$, because it is completely arbitrary. And this is precisely the knot topology of the QCD vacuum [25].

This is not surprising. Given the fact that there is a deep connection between Skyrme theory and QCD, it is natural that the Skyrme theory and QCD have similar vacuum structure. To amplify this point, notice that the most general SU(2) QCD vacuum can be expressed in terms of a right-handed $S U(2)$ basis $\left(\hat{n}_{1}, \hat{n}_{2}, \hat{n}_{3}=\hat{n}\right)$ by [25]

$$
\hat{\Omega}_{\mu}=-\frac{1}{2} \epsilon_{i j k}\left(\hat{n}_{i} \cdot \partial_{\mu} \hat{n}_{j}\right) \hat{n}_{k} .
$$

Clearly it has the $\pi_{3}\left(S^{3}\right)$ topology of the mapping from the compactified 3-dimensional space to the SU(2) group space defined by $\left(\hat{n}_{1}, \hat{n}_{2}, \hat{n}\right)$. But since $\left(\hat{n}_{1}, \hat{n}_{2}, \hat{n}\right)$ is completely determined by $\hat{n}$ up to the $\mathrm{U}(1)$ rotation which leaves $\hat{n}$ invariant, (30) also has the knot topology $\pi_{3}\left(S^{2}\right)$ which describes the mapping from the real space $S^{3}$ to the coset space $S^{2}$ of $\mathrm{SU}(2) / U(1)$.

Now it must be clear why the vacuum of Skyrme theory has the same knot topology. As we have noticed, the Skyrme theory has the vacuum (29) independent of $\hat{n}$, and this $\hat{n}$ adds the knot topology $\pi_{3}\left(S^{2}\right)$ to the vacuum. Of course, in the Skyrme theory we do not need the vacuum potential (30) to describe the vacuum. We only need $\hat{n}$ which describes the knot topology.

This confirms that the vacuum in Skyrme theory has the topology of the Sine-Gordon theory and QCD combined together. This means that the vacuum of the Skyrme theory can also be classified by two quantum numbers $(p, q)$, the $\pi_{1}\left(S^{1}\right)$ of $\omega$ and $\pi_{3}\left(S^{2}\right)$ of $\hat{n}$. And this is so without any extra potential. As far as we know, there is no other theory which has this type of vacuum topology.

At this point we emphasize the followings. First, the knot topology of $\hat{n}$ is different from the monopole topology of $\hat{n}$. The monopole topology $\pi_{2}\left(S^{2}\right)$ is associated to the isolated singularities of $\hat{n}$, but the knot topology $\pi_{3}\left(S^{2}\right)$ does not require any singularity for $\hat{n}$. And for a classical vacuum $\hat{n}$ must be completely regular everywhere. So only the knot topology, not the monopole topology, can not describe a classical vacuum. And this is precisely the vacuum topology of QCD. 
Second, the knot topology of the vacuum is different from the Faddeev-Niemi knot that we have in the Skyrme theory [21]. The Faddeev-Niemi knot is a unique and real (i.e., physical) knot which carries energy, which is given by the solution of (17). In particular, we have the knot solution when $\omega=(2 n+1) \pi$. On the other hand, we have the knot of the vacuum when $\omega=2 \pi p$. Moreover, the vacuum knot has no energy, and is not unique. While the Faddeev-Niemi knot is unique, there are infinitely many $\hat{n}$ which describes the same vacuum knot topology. So obviously they are different. What is really remarkable is that the same $\hat{n}$ has multiple roles. It describes the monopole topology, the knot topology of Faddeev-Niemi knot, and the knot topology of the vacuum.

\section{Discussions}

Skyrme theory has been known to have rich topological structures. It has the Wu-Yang type monopoles, the skyrmions as dressed monopoles, the baby skyrmions and twisted magnetic flux, and the Faddeev-Niemi knots made of twisted magnetic vortex ring [21-23]. This makes the theory very important not only in high energy physics but also in condensed matter physics, in particular in two-gap superconductor and two-component Bose-Einstein condensates [22, 23, 26-29].

Our analysis tells that the theory has more topology. In this paper we have shown that the skyrmions are not just the dressed monopoles but actually carry the monopole number, so that they can be classified by two topological numbers, the baryon number and the monopole number. Moreover, we have shown that here the baryon number could be replaced by the radial (shell) number, so that the skyrmions can be classified by two topological numbers $(m, n)$, the monopole number $m$ which describes the $\pi_{2}\left(S^{2}\right)$ topology of the $\hat{n}$ field and the radial (shell) number $n$ which describes the $\pi_{1}\left(S^{1}\right)$ topology of the $\omega$ field. In this scheme the baryon number $B$ is given by the product of two integers $B=m n$. This comes from the following facts. First, the SU(2) space $S^{3}$ admits the Hopf fibering $S^{3} \simeq S^{2} \times S^{1}$. Second, the Skyrme theory has two variables, the angular variable $\omega$ which can represent the $\pi_{1}\left(S^{1}\right)$ topology and the coset variable $\hat{n}$ which represents the $\pi_{2}\left(S^{2}\right)$ topology.

In this view the popular (non spherically symmetric) skyrmions are classified as the $(m, 1)$ skyrmions, and the radially excited spherically symmetric skyrmions are classified as the $(1, n)$ skyrmions. and we can construct the $(m, n)$ skyrmions adding the shell structure to the $(m, 1)$ skyrmions. Moreover, we have shown that the skyrmions, when they are generalized to have two topological numbers, should have the topological stability of the two topology independently. This is remarkable.
As importantly, we have shown that the Skyrme theory has multiple vacua. The vacuum of the theory has the structure of the vacuum of the Sine-Gordon theory and at the same time the structure of QCD vacuum. So the vacuum can also be classified by two topological numbers $p$ and $q$ which represent the $\pi_{1}\left(S^{1}\right)$ topology of the $\omega$ field and the $\pi_{3}\left(S^{2}\right)$ topology of the $\hat{n}$ field.

The fact that the vacuum of the Skyrme theory has the $\pi_{1}\left(S^{1}\right)$ topology is not surprising, considering that it has the angular variable $\omega$. Moreover, the fact that the vacuum of the Skyrme theory has the $\pi_{3}\left(S^{2}\right)$ topology of the QCD vacuum could easily be understood once we understand that the Skyrme theory is closely related to QCD. What is really remarkable is that it has both $\pi_{1}\left(S^{1}\right)$ and $\pi_{3}\left(S^{2}\right)$ topology at the same time. As far as we understand there is no other theory which has this feature. This again is closely related to the fact that $S^{3}$ admits the Hofp fibering and that the theory has two variables $\omega$ and $\hat{n}$.

This raises interesting questions. Can we generalize the Faddeev-Niemi knot to have the $\pi_{1}\left(S^{1}\right)$ topology? If so, how do we obtain such knot? Do we have the vacuum tunneling in Skyrme theory? What instanton can we have in this theory?

Clearly the above observations put the Skyrme theory in a totally new perspective. Our results in this paper show that the theory has so many new aspects which make the theory more interesting. But most importantly our results strongly imply that we need a new interpretation of the Skyrme theory.

Note Added One of the referees suggested that there might be a strong similarity between the Hopf map $S^{3} \rightarrow S^{2} \rightarrow S^{2}$ discussed by Adam et al. [30,31] and our result that the baryon number could be decomposed to the monopole number and the shell number. Although there is no direct relation between this work and our result, the Hopf fibering $S^{3} \simeq S^{2} \times S^{1}$ does play the central role for us to justify the existence of the shell number, as we have emphasized in this paper. The details of the above results and the questions raised in this paper will be discussed in a separate publication [32].

Acknowledgements The work is supported in part by the National Natural Science Foundation of China (Grant 11575254), Chinese Academy of Sciences Visiting Professorship for Senior International Scientists (Grant 2013T2J0010), National Research Foundation of Korea (Grants 2015-R1D1A1A01-057578 and 2015-R1D1A1A01-059407), and by Konkuk University.

Open Access This article is distributed under the terms of the Creative Commons Attribution 4.0 International License (http://creativecomm ons.org/licenses/by/4.0/), which permits unrestricted use, distribution, and reproduction in any medium, provided you give appropriate credit to the original author(s) and the source, provide a link to the Creative Commons license, and indicate if changes were made. Funded by SCOAP $^{3}$. 


\section{References}

1. T.H.R. Skyrme, Proc. Roy. Soc. (London) 260, 127 (1961)

2. T.H.R. Skyrme, Proc. Roy. Soc. (London) 262, 237 (1961)

3. T.H.R. Skyrme, Nucl. Phys. 31, 556 (1962)

4. G. Adkins, C. Nappi, E. Witten, Nucl. Phys. B 228, 552 (1983)

5. A. Jackson, M. Rho, Phys. Rev. Lett. 51, 751 (1983)

6. E.B. Bogomolny, V.A. Fateev, Sov. J. Nucl. Phys. 37, 134 (1983)

7. See, for example, I. Zahed and G. Brown, Phys. Rep. 142, 1 (1986), and references therein

8. N.S. Manton, Phys. Lett. B 192, 177 (1987)

9. R.A. Battye, P.M. Sutcliffe, Phys. Rev. Lett. 79, 363 (1997)

10. C.J. Houghton, N.S. Manton, P.M. Sutcliffe, Nucl. Phys. B 510, 507 (1998)

11. N.S. Manton and B. Piette, in Proceedings of the European Congress of Mathematics, Barcelona (2000), edited by C. Casacuberta et al., Progress in Mathematics Vol. 201 (Birkhauser, Basel, 2001)

12. R.A. Battye, P.M. Sutcliffe, Phys. Rev. Lett. 86, 3989 (2001)

13. R.A. Battye, P.M. Sutcliffe, Rev. Math. Phys. 14, 29 (2002)

14. D.T.J. Feist, P.H.C. Lau, N.S. Manton, Phys. Rev. D 87, 085034 (2013)

15. F. Hoyle, Astrophys. J. Suppl. 1, 121 (1954)

16. E. Epelbaum, H. Krebs, D. Lee, U. Meissner, Phys. Rev. Lett. 106, $192501(2011)$
17. P.H.C. Lau, N.S. Manton, Phys. Rev. Lett. 113, 232503 (2014)

18. C.J. Halcrow, N.S. Manton, JHEP 1501, 016 (2015)

19. C. Adam, J. Sanches-Guillen, A. Wereszczynski, Phys. Lett. B 691, 105 (2010)

20. C. Adam, J. Sanches-Guillen, A. Wereszczynski, Phys. Rev. Lett. 111, 232501 (2013)

21. Y.M. Cho, Phys. Rev. Lett. 87, 252001 (2001)

22. Y.M. Cho, Phys. Lett. B 603, 88 (2004)

23. Y.M. Cho, B.S. Park, P.M. Zhang, Int. J. Mod. Phys. A 23, 267 (2008)

24. S.B. Gudnason, M. Nitta, Phys. Rev. D 90, 085007 (2014)

25. Y.M. Cho, Phys. Lett. B 644, 208 (2007)

26. J. Ruostekoski, J.R. Anglin, Phys. Rev. Lett. 86, 3934 (2001)

27. R.A. Battye, N.R. Cooper, P.M. Sutcliffe, Phys. Rev. Lett. 88, 080401 (2002)

28. Y.M. Cho, H. Kim, P. Zhang, Phys. Rev. A 72, 063603 (2005)

29. Y.M. Cho, P.M. Zhang, Eur. Phys. J. B 65, 155 (2008)

30. C. Adam, B. Muratori, C. Nash, Phys. Lett. B 479, 329 (2000)

31. C. Adam, B. Muratori, C. Nash, Phys. Rev. D 61, 105108 (2000)

32. P. Zhang, K. Kimm, J.H. Yoon, Y.M. Cho. to be published 